\title{
Isospin Effects in Nuclear Fragmentation
}

\author{
V.Baran ${ }^{1,2}$, M.Colonna ${ }^{1}$, M.Di Toro ${ }^{1}$, V.Greco ${ }^{1}$ \\ M.Zielinska-Pfabé ${ }^{3}$ and H.H.Wolter ${ }^{4}$ \\ 1) Laboratori Nazionali del Sud, Via S. Sofia 44, I-95123 Catania, Italy \\ and University of Catania \\ 2) NIPNE-HH, Bucharest, Romania \\ 3) Smith College, Northampton, USA \\ 4) Sektion Physik, University of Munich, Germany
}

\begin{abstract}
We investigate properties of the symmetry term in the equation-of-state (EOS) of nuclear matter (NM) from the analysis of simulations of fragmentation events in intermediate energy heavy ion collisions. For charge asymmetric systems a qualitative new feature in the liquid-gas phase transition is predicted: the onset of chemical instabilities with a mixture of isoscalar and isovector components. This leads to a separation into a higher density ("liquid") symmetric and a low density ("gas") neutron-rich phase, the so-called neutron distillation effect. We analyse the simulations with respect to the time evolution of the isospin dynamics, as well as with respect to the distribution and asymmetry of the final primary fragments. Qualitatively different effects arise in central collisions, with bulk fragmentation, and peripheral collisions with neck-fragmentation. The neck fragments produced in this type of process appear systematically more neutron-rich from a dynamical nucleon migration effect which is very sensitive to the symmetry term in regions just below normal density.

In general the isospin dynamics plays an important role in all the steps of the reaction, from prompt nucleon emission to the sequential decay of the primary fragments. A fully microscopic description of the reaction dynamics including stochastic elements to treat fluctuations realistically is absolutely necessary in order to extract precise information on the fragmentation and the nuclear equation of state. We have performed simulations for fragment production events in $n$-rich $\left({ }^{124} S n\right)$ and $n$-poor $\left({ }^{112} \mathrm{Sn}\right)$ symmetric colliding systems. We test the dependence of the isospin dynamics on the isospin EOS and on the neutron enrichment of the system.
\end{abstract}

Key words: Asymmetric nuclear matter, Isospin dynamics, Fragmentation PACS: 21.65.+f, 25.70.Pq, 25.70.Mn, 24.10.Cn 


\section{Introduction}

The availability of exotic (radioactive) beams has driven a strong interest in nuclear structure studies of $\beta$-unstable nuclei. It is clear that essential complementary information will come from charge asymmetry effects on nonequilibrium nuclear dynamics of heavy ion collisions. A quantitative analysis of this aspect, trying to identify some sensitive observables in the reaction mechanisms, is the aim of the present work.

We will show that it should be possible to extract information on the symmetry term of the nuclear EOS in regions away from normal density under laboratory controlled conditions. Asymmetric nuclear matter models at high density have been tested so far only in astrophysics contexts, in particular in supernovae explosions and neutron stars [1-6]. Although in heavy ion collisions at intermediate energies we certainly cannot reach very high density regions, we should be able to obtain information on the slope of the symmetry term, i.e. the Symmetry Pressure, below the saturation density. We then can put experimental constraints on the effective interactions used in astrophysical contexts $[7,8]$. Moreover we like to remark that the same symmetry pressure is of relevant importance for structure properties, being clearly linked to the thickness of the neutron skin in n-rich (stable and/or unstable) nuclei (see [9] and the discussion in refs. $[10,11])$.

There are very stimulating predictions on new phases of asymmetric nuclear matter that eventually could be reached in heavy ion reaction dynamics with radioactive beams. The onset of coupled mechanical and chemical instabilities is envisaged [12-15], that should lead to interesting experimental signatures of the dynamics of this new phase transition. In particular, compared to statistical models more symmetric and narrower isotopic distributions of primary fragments are predicted, which should be sensitive to the symmetry term of the EOS. For semi-peripheral collisions the isospin dynamics of the participant zone is seen to be affected by the symmetry term [16] with respect to neck-dynamics, mid-rapidity fragmentation and fast-fission of the spectators [17-21]. We will see that independent new information on the symmetry term can be derived.

In the last years some first data have appeared on isospin effects in reaction dynamics with a few theoretical analyses (see the recent reviews [22-25]). Although the data are mostly of inclusive type and the theoretical studies have not focussed on the effect of different symmetry terms, a noticeable dependence of the reaction mechanism on the charge asymmetry emerges clearly. Very recently accurate results from high-performance $4 \pi$-detectors have appeared [26-32] that are strongly stimulating theoretical interpretations [33,34]. These are the main motivations of this work. 
In section 2 we discuss the wide range of predictions that still exist on the density dependence of the symmetry term in nuclear matter. Section 3 reviews the results on new features of the liquid-gas phase transition in asymmetric $\mathrm{NM}$ and the isospin distillation effect. In sect. 4 the isospin dependent transport equations for the collision dynamics are introduced with special attention to the construction of the stochastic term. Section 5 is devoted to a detailed discussion of the "ab initio" collision simulations for $n$-rich and $n$-poor systems using different equations of state. A summary of the main results with related perspectives is given in Section 6 .

\section{Symmetry term effects on compressibility, saturation density and the nucleon mean field}

A key question in the physics of unstable nuclei is the knowledge of the EOS for asymmetric nuclear matter away from normal conditions. We recall again that the symmetry term at low densities affects the neutron skin structure, while in the high density region it is crucial for supernovae dynamics and neutron star cooling. The paradoxial situation is that while we are planning second and third generation facilities for radioactive beams our basic knowledge of the symmetry term of the EOS is still extremely poor. Effective interactions are obviously tuned to symmetry properties around normal conditions and extrapolations are very uncertain. Microscopic approaches based on realistic NN interactions, Brueckner or variational schemes, or on effective field theories show a large range of predictions. As an example, in fig. 1 we show the isospin dependence of some EOS's which, however, have the identical saturation properties for symmetric NM: $S K M^{*}$ [35], SLy230b (SLy4) [36] and BPAL32 [7,37].

In the following we will refer to an "asy-stiff" EOS when we are considering a potential symmetry term linearly increasing with nuclear density and to a "asy-soft" EOS when the symmetry term shows a saturation and sometimes even a decrease at higher densities [24]. In some cases, in order to enhance the dynamical effects, we will consider also an "asy-superstiff" behaviour with a roughly parabolic increase of the symmetry term with density $[8,33,37]$. In the nuclear equation of state the symmetry term is written in the form

$$
\frac{E}{A}(\rho, I)=\frac{E}{A}(\rho)+\frac{E_{\text {sym }}}{A}(\rho) I^{2}
$$

with $I=(N-Z) / A$ the asymmetry parameter. The kinetic and potential 


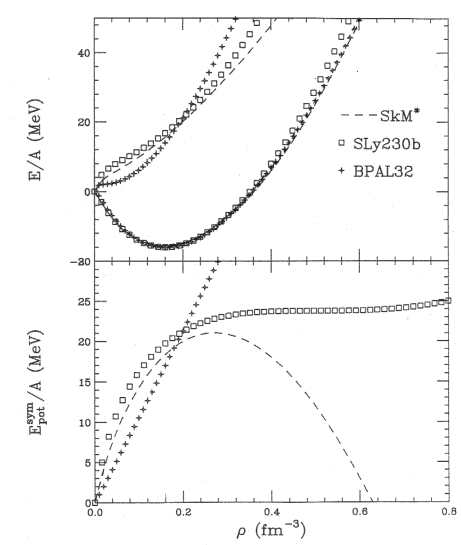

Fig. 1. EOS for various effective forces. Top panel: neutron matter (upper curves), symmetric matter (lower curves); Bottom panel: symmetry term of potential energy. contributions are

$$
\epsilon_{\text {sym }} \equiv \frac{E_{\text {sym }}}{A}(\rho)=\frac{\epsilon_{F}(\rho)}{3}+\frac{C(\rho)}{2} \frac{\rho}{\rho_{0}}
$$

In a Skyrme-like parametrization of effective interactions the function $C(\rho)$ has the form:

$$
\begin{aligned}
\frac{C(\rho)}{\rho_{0}}= & -\frac{1}{4}\left[t_{0}\left(1+2 x_{0}\right)+\frac{t_{3}}{6}\left(1+2 x_{3}\right) \rho^{\alpha}\right]+ \\
& \frac{1}{12}\left[t_{2}\left(4+5 x_{2}\right)-3 t_{1} x_{1}\right]\left(\frac{3 \pi^{2}}{2}\right)^{2 / 3} \rho^{2 / 3}
\end{aligned}
$$

We note that the second term is related to isospin effects in the momentum dependence [36].

In fig.1 (bottom) we report the density dependence of the potential symmetry contribution, i.e. the second term of eq.(2), for the three different effective interactions shown in the upper panel. While all curves obviously cross at normal density $\rho_{0}$, quite large differences are seen with repect to values and 
slopes in low density and particularly in high density regions. We note especially the uncertainty on the symmetry pressure even around $\rho_{0}$, which is of great importance for structure calculations, as mentioned in the introduction.

We start to discuss some simple considerations of asymmetry effects in infinite matter on compressibility and saturation density, observables related to monopole resonances and to the bulk density in a heavy nucleus [38]. From a linear expansion of the energy around the symmetry value we obtain for the variation of saturation density with asymmetry

$$
\Delta \rho_{0}(I)=-\left.\frac{9 \rho_{0}^{2}}{K_{N M}(I=0)} \frac{d}{d \rho} \frac{E_{s y m}}{A}(\rho)\right|_{\rho=\rho_{0}} I^{2}<0,
$$

where $K_{N M}(I=0)$ and $\rho_{0}$ are respectively compressibility and saturation density of symmetric NM. Eq.(4) has an intuitive geometrical interpretation. Asymmetry results in an extra pressure $P_{\text {sym }}=\rho^{2} d \epsilon_{\text {sym }} / d \rho$ that can be compensated by moving to the left the saturation point $(P=0)$ by the quantity $\Delta \rho_{0}$. For the compressibility shift we have, after some algebra,

$$
\Delta K_{N M}(I)=\left.9 \rho_{0}\left[\rho_{0} \frac{d^{2}}{d \rho^{2}}-2 \frac{d}{d \rho}\right] \frac{E_{\text {sym }}}{A}(\rho)\right|_{\rho=\rho_{0}} I^{2}<0
$$

to note the interplay between slope and curvature of the symmetry term.

The predictions are quite different even for relatively small asymmetries. E.g., as expected from fig.1, $S K M^{*}$ gives the largest variation for the compressibility and the smallest for the saturation density [38]. Thus there are good chances of a direct experimental observation.

From eqs. (1-3) we can derive a general Skyrme-like form for neutron and proton mean field potentials

$$
U_{q}=A\left(\frac{\rho_{s}}{\rho_{0}}\right)+B\left(\frac{\rho_{s}}{\rho_{0}}\right)^{\alpha+1}+C\left(\frac{\rho_{i}}{\rho_{0}}\right) \tau_{q}+\frac{1}{2} \frac{\partial C}{\partial \rho_{s}} \frac{\rho_{i}^{2}}{\rho_{0}}
$$

where $\rho_{s} \equiv \rho_{n}+\rho_{p}$ and $\rho_{i} \equiv \rho_{n}-\rho_{p}$ are respectively isoscalar and isovector densities, and $\tau_{q}=+1(q=n)$, respectively $\tau_{q}=-1(q=p)$. The symmetry contribution to the mean field (second part of eq.(6)) for the parametrizations discussed in this work has the following form: For the "asy-soft" EOS we use the $S K M *$ parametrization shown in fig. 1. For the "asy-stiff" EOS the density dependence is given by $C(\rho)=$ const $\simeq 32 \mathrm{MeV}$. For the "asy-superstiff" EOS we use a symmetry term rapidly increasing around normal density with a form $2 \rho^{2} / \rho_{0}\left(\rho+\rho_{0}\right)[33,37]$. The density dependence of the neutron and proton mean fields for these parametrizations are shown in Fig.2 for a system with $N=1.5 Z(I=0.2)$. 


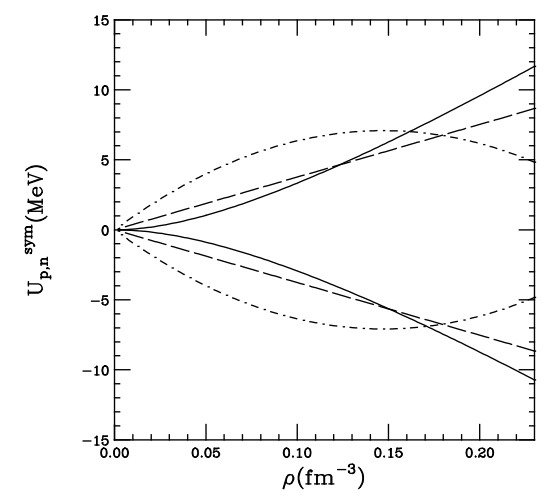

Fig. 2. Symmetry contribution to the mean field at $I=0.2$ for neutrons (upper curves) and protons (lower curves): dashed lines "asy-soft", solid lines "asy-stiff", long dashed lines "asy-superstiff"

In regions just off normal density the field "seen" by neutrons and protons in the three cases is very different, especially below normal density. We thus expect important transport effects during the reaction at intermediate energies since the interacting asymmetric nuclear matter will experience compressed and expanding phases before forming fragments around normal density conditions.

A complementary and maybe more complete picture of the isospin dynamics can be obtained from the analysis of the density dependence of the neutron/proton chemical potentials $\mu_{q} \equiv \partial \epsilon\left(\rho_{q}, \rho_{q^{\prime}}\right) / \partial \rho_{q}, \epsilon$ being the energy density. We recall that the chemical potentials contain all the contributions to the energy per particle, including the scalar part and the kinetic symmetry term in asymmetric matter. In this sense their study is quite natural for the energetic arguments, while the symmetry mean field of fig. 2 , although very instructive, shows only the potential part. We note that in non-equilibrium processes the mass flow is determined by the differences in the local values of chemical potential and it is directed from the regions of higher chemical potential to regions of lower values until equalization. This is analogous to 
what happens with the heat in a temperature gradient. For a two component systems such discussion should be performed for each species.

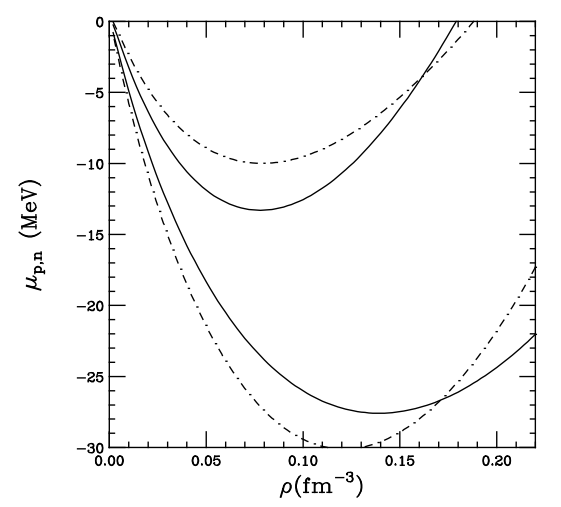

Fig. 3. Density dependence of proton (lower curves) and neutron (upper curves) chemical potentials for an asy-superstiff (solid lines) and asy-soft (dashed line) EOS for asymmetry $I=0.2$.

In fig. 3 we show the proton and neutron chemical potentials for symmetric and asymmetric $(I=0.2)$ nuclear matter for two isospin EOS (soft, superstiff). We note that the difference between neutron and proton chemical potentials is given by the relation

$$
\mu_{n}-\mu_{p}=4 \frac{E_{s y m}}{A} I
$$

It thus reflects directly the density dependence of the symmetry potential as seen in the lower panel of Fig. 1. Two regions with significantly different behaviour can be distinguished below normal density. In the region $\rho \leq .08 \mathrm{fm}^{-3}$ neutrons and protons will move both to higher densities, while in the region above and up to density $\rho_{0}$ neutrons will move to lower and proton to higher densities. This will be seen to be useful for the interpretation of the reaction dynamics. 


\section{Mechanical and Chemical Instabilities in Dilute Nuclear Matter: Isospin Distillation}

For charge asymmetric systems we expect qualitatively new features in the liquid-gas phase transition; the onset of chemical instabilities that will show up in a novel nature of the unstable modes, given by a mixture of isoscalar and isovector components. In this section we will review the results in nuclear matter as given in refs. $[12,15]$. In the framework of Landau theory for two component Fermi liquids the spinodal border is determined by studying the stability of collective modes described by two coupled Landau-Vlasov equations for protons and neutrons. In terms of the appropriate Landau parameters the stability condition can be expressed as [41]

$$
\left(1+F_{0}^{n n}\right)\left(1+F_{0}^{p p}\right)-F_{0}^{n p} F_{0}^{p n}>0
$$

It was shown in [15] that this condition is equivalent to the following thermodynamical condition

$$
\left(\frac{\partial P}{\partial \rho}\right)_{T, y}\left(\frac{\partial \mu_{p}}{\partial y}\right)_{T, P}>0
$$

discussed in $[12,15,42]$, where $y$ is the proton fraction and $\mu_{p}$ the proton chemical potential. In fig. 4 we show the spinodal boundaries obtained from eq. (8) (continuous line with circles). The calculations are performed with the non-relativistic Skyrme-like force $S K M^{*}$ [15], but very similar results can be obtained with relativistic mean field approaches [12]. For asymmetric nuclear matter these boundaries are seen to contain the region corresponding to "mechanical instability", $\left(\frac{\partial P}{\partial \rho}\right)_{T, y}<0$ (crosses). Outside, in the area between crosses and circles, the instability of the system is thus driven by the chemical condition $\left(\frac{\partial \mu_{p}}{\partial y}\right)<0$.

We note, however, that just from the above stability conditions we cannot determine the nature of the fluctuations against which a binary system becomes chemically unstable. Indeed, the thermodynamical condition in eq. (9) cannot distinguish between two very different situations which can be encountered in nature: an attractive interaction between the two components of the mixture $\left(F_{0}^{n p}, F_{0}^{p n}<0\right)$, as is the case of nuclear matter, or a repulsive interaction between the two species. We define density fluctuations as isoscalar-like in the case when proton and neutron Fermi spheres (or equivalently the proton and neutron densities) fluctuate in phase and as isovector-like when the two Fermi spheres fluctuate out of phase. Then it was shown, based on a thermodynamical approach to asymmetric Fermi liquid mixtures [15], that chemical instabilities are triggered by isoscalar-like fluctuations in the first, i.e. attrac- 
tive, situation and by isovector-like fluctuations in the second one. For the dilute asymmetric nuclear matter case, because of the attractive interaction between protons and neutrons at low density, the phase transition is thus due to isoscalar-like fluctuations that induce chemical instabilities while the system is never unstable against isovector fluctuations.

The apparent paradox that chemical instabilities are due to isoscalar-like fluctuations can be understood from the behaviour of the chemical potenials in fig. 3. In the low-density region, where the instability occurs, both proton and neutron chemical potentials decrease with density thus leading to an isoscalarlike fluctuation. However, the slope of the chemical potentials is different for protons and neutrons, thus leading to a more proton-rich denser phase ("liquid") and a "chemical" effect, the neutron distillation.

Of course the same attractive interaction is also at the origin of phase transitions in symmetric nuclear matter. However, in the asymmetric case isoscalar fluctuations lead to a more symmetric high density phase everywhere under the instability line defined by the eqs. $(8,9) \quad[15,41]$ and therefore to a more neutron-rich gas (isospin distillation). The mechanical instability zone shown in figs.3b,3c for asymmetric cases, has not a real physical meaning: the same isospin distillation effect also happens if the system is prepared inside the mechanical instability region. Thus there is a smooth transition from "chemical" to "mechanical" instability [15].

Since during the dynamics of a collision the system can deeply enter the instability region it is important to have a more detailed information on the space-time structure of the unstable modes leading to fragment formation. This can be discussed in the Landau kinetic approach to the linear response theory $[42,43]$. The Landau dispersion relations have indeed imaginary sound velocity solutions with well defined structure in the $\left(\delta \rho_{n}, \delta \rho_{p}\right)$ space $[14,41]$. They always lead to the prediction of a very neutron rich gas phase versus a more symmetric liquid phase formed in a dynamical non-equilibrium mechanism on short time scales. This "chemical effect" is very sensitive to the symmetry term of the effective interaction below saturation density [14], thus providing a good opportunity to distinguish between different isospin EOS.

In equilibrium statistical multifragmentation calculations [44] sensitive observables appear to be the yield ratios of light isobars (e.g. $t /{ }^{3} H e$ ) and the formation of more stable primary intermediate mass fragments [45]. Therefore in the following, from "ab initio" dynamical simulations of central collisions, we will discuss the effects of the structure of the instabilities on fragment observables (mass/charge-yields, isospin content and multiplicity distributions) and on the reaction mechanism, i.e. the time evolution of the isospin dynamics. Complementary information will be obtained from the comparison of bulk and neck fragmentation events in semi-central and semi-peripheral collisions, 

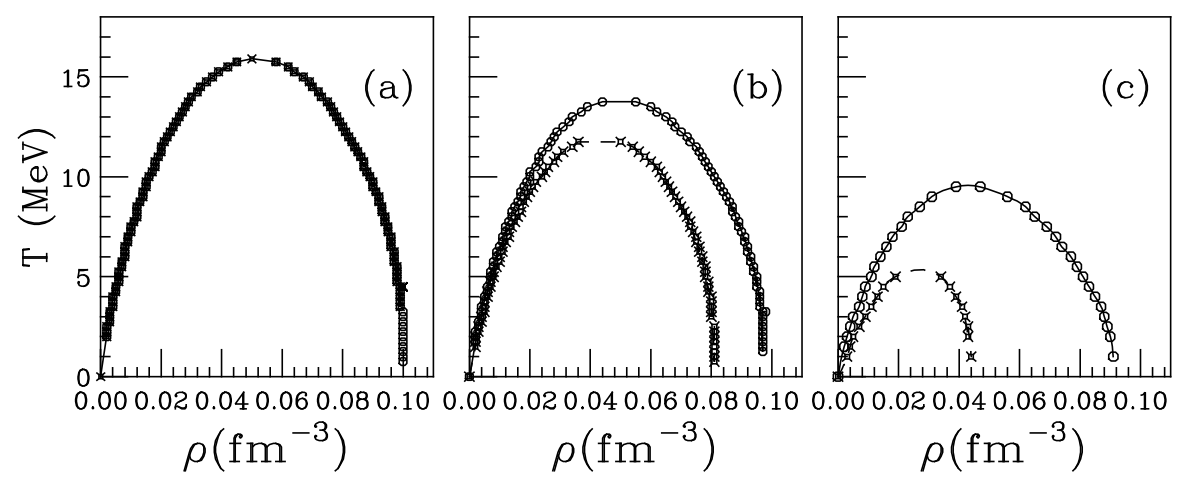

Fig. 4. Spinodal boundary of asymmetric nuclear matter (open circles) and mechanical instability boundary (crosses) for three proton fractions: a) $y=0.5, b) y=0.25$, c) $y=0.1$

where the latter represent a large part of the reaction cross section [17].

\section{Stochastic Transport Simulations}

A new code for the solution of microscopic transport equations of BoltzmannNordheim-Vlasov $(B N V)$ type [46-50] has been written where asymmetry effects are suitably accounted for $[16,51]$ and where fluctuations are included dynamically [52]. A density dependent symmetry term is used consistently in the construction of the initial ground state and the evolution of the reaction, isospin effects on nucleon cross sections and Pauli blocking are consistently evaluated. The transport equations are solved following a test particle evolution on a lattice $[50,53]$. A parametrization of free NN cross sections is used with energy and angular dependence.

It is important to include fluctuations when discussing dynamical fragment formation. Indeed, the evolution under the influence of fluctuations is described by a transport equation with a stochastic fluctuating term, the so-called Boltzmann-Langevin equation [54-56]. In this paper we will follow two methods to include fluctuations, the Stochastic Mean Field method[52] and the cor- 
responding simplified approach used in [57-59]. The Stochastic Mean Field method is based on a fully self-consistent treatment of the fluctuations during the time evolution. On the path towards local thermal equilibrium, the system is characterized by a mean trajectory $\bar{f}$ and a variance $\sigma^{2}=<(f-\bar{f})^{2}>$ which in each phase space cell obeys the equation of motion

$$
\frac{d}{d t} \sigma^{2}=-\frac{2}{\tau(t)} \sigma^{2}+2 D(t)
$$

with $2 D(t)$ the correlation function of the fluctuating term and $\tau(t)=1 /\left(w^{+}+\right.$ $w^{-}$), where $w^{+}$and $w^{-}$are the transition probabilities into and out of the phase space cell. The equilibrium statistical value $\sigma_{0}^{2}=f_{0}\left(t_{e q}\right)\left(1-f_{0}\left(t_{e q}\right)\right)$ suggests an ansatz for the correlation function of the fluctuation term of the form

$$
2 D(t)=(1-\bar{f}) w^{+}+\bar{f} w^{-}
$$

i.e. the magnitude of the fluctuations is given by the total number of collisions (fluctuation-dissipation theorem) [55]. Then we obtain for the time evolution of the quantity $\Delta=\sigma^{2}-\bar{f}(1-\bar{f})$

$$
\frac{d}{d t} \Delta=-\frac{2}{\tau(t)} \Delta
$$

$\Delta=0$ is a solution of eq.(12). Therefore, if the variance is initially locally set equal to its statistical value, it will always be given by the local statistical variance as $\sigma^{2}=\bar{f}(1-\bar{f})$. With a projection of this relation on coordinate space we obtain local density fluctuations which are implemented with a MonteCarlo method at each time step. In this way we also have a branching of trajectories. The procedure is valid if we assume a local statistical equilibrium, appropriate for the problems discussed here, i.e. fragment production in the expansion/separation phase.

The other approach, computationally much easier [57-59], is based on the introduction of density fluctuations by a random sampling of the phase space. The amplitude of the noise is gauged to reproduce the dynamics of the most unstable modes [58]. For each system we have checked the equivalence of the two methods in the description of the collision dynamics in the complete evolution from fast particle emissions to the fragment production. The analysis of the results, presented in the next Section, is based on events collected with both methods. In the implementations isospin effects in the fluctuations have been consistently accounted for. 


\section{Dynamical Simulations}

In this section we want to show the possibility of extracting quantitative information on the symmetry term of the EOS directly from fragmentation reactions using stable isotopes with different charge asymmetries. The first exclusive data for such reactions are now starting to become available [26-32]. We study neutron-rich and neutron-poor $S n+S n$ collisions. In spite of the relatively low asymmetries tested with stable isotopes we will see an interesting and promising dependence on the stiffness of the symmetry term.

The dynamics of a fragmentation reaction is extremely rich. The time evolution can be roughly divided into the following phases: pre-equilibrium particle emission, compression-expansion stage, multifragmentation ending in the freeze-out configuration, and statistical decay of the primary fragments. All the steps are seen to be very isospin dependent and therefore it is essential to perform consistent "ab initio" simulations of the whole process in order to extract information on the symmetry term of the EOS. Since in the reaction dynamics with intermediate energy beams the asymmetric nuclear matter probes compressed as well as dilute phases the final output will be determined by the complete density dependence of symmetry term. The aim of this paper is to show which fragmentation observables are particularly sensitive to the symmetry term of the EOS. We will see that the physical interpretation of the results can be made quite transparent in spite of the complexity of the numerical simulations. The new features of the liquid-gas phase transition in asymmetric nuclear systems discussed in the previous sections were based on thermodynamical considerations [12,13,15], linear response approaches [14] or dynamical simulations in a box [41] (i.e. without finite size and Coulomb effects). It is important to check these predictions in realistic simulations of fragmentation reactions for systems with different charge asymmetries.

In order to simplify the analysis of the most sensitive observables to isospin effects we have chosen a Skyrme force with the same soft EOS for symmetric NM $(K=201 M e V)$ and with three different choices for the density dependence of the symmetry term, i.e. the asy-soft, stiff, and superstiff interactions discussed in sect. 2, and qualitatively seen in fig. 2 . In this way we force the symmetric part of the EOS to be exactly the same in order to disentangle dynamical symmetry term effects. As we see in fig. 2 the potential symmetry term for the various interactions shows quite different behaviours in the region around normal density and at very low densities. While around $\rho_{0}$ the density dependence becomes steeper when we go from asy-soft to asy-superstiff (this suggests the names) at subsaturation densities, where we enter the spinodal zone and the fragment formation initiates, they manifest an opposite trend. We will show that the reaction mechanism is sensitive to these different behaviours, even though with stable nuclei we are limited in the possible 
asymmetries to be explored (and moreover we will certainly not reach high compression regions).

\subsection{Reaction Mechanisms}

We have studied collisions of the systems ${ }^{124} S n+{ }^{124} S n$ and ${ }^{112} S n+{ }^{112} S n$ at $50 \mathrm{AMeV}$, where new data have just appeared or are under analysis at MSU [26-28]. We investigate semi-central $(b=2 \mathrm{fm})$ and semi-peripheral $(b=6 \mathrm{fm})$ reactions for each of the three isospin EOS. The analyses are based on around 500 events in each case. In order to get a qualitative impression of the reaction mechanism the time evolution of the density (projected on the reaction plane) is shown in figs. 5 and 6 for impact parameters $b=2$, 4 , and $6 \mathrm{fm}$ for one typical event each (neutron rich case, asy-stiff EOS).

For a semi-central collision, $b=2 \mathrm{fm}$, the reaction mechanism corresponds to bulk fragmentation. We can identify three main stages that are characterized by specific features of the isospin dynamics since the system explores different density regions. After a first compression phase (until about $40-50 \mathrm{fm} / \mathrm{c}$ ) a fast expansion phase follows (until 110-120 $\mathrm{fm} / \mathrm{c}$ ). Then during the fragmentation stage the system will break up and the fragment formation process takes place up to the freeze-out time (around 260-280 fm/c). At this time excited primary fragments have been formed which are far enough apart to have a negligible mutual nuclear interaction (see fig.4). This freeze-out time is relatively well defined in the simulations as the time when the average number of produced fragments stabilizes. The physical conditions of density and temperature at the beginning of the fragmentation stage correspond to an unstable nuclear matter phase. The volume instabilities have time to develop and we expect to see a spinodal decomposition with the formation of a liquid and a gas phase.

For $b=6 \mathrm{fm}$, (semi-peripheral collision, fig. 6) we observe quite a different behaviour. Now in the overlap region a neck structure is developing. During the interaction time (from about 80 to $120 \mathrm{fm} / \mathrm{c}$ ) it heats and expands but remains in contact with the denser and colder regions of the projectile-like (PLF) and/or target-like (TLF) fragments. Now the surface instabilities of a cylindrically shaped neck region and the fast leading motion of the PLF and TLF will play the important role. At the freeze-out time with the neck rupture at about $140 \mathrm{fm} / \mathrm{c}$ intermediate mass fragments (IMF) are produced in the mid-rapidity zone. One can have a large variety of event structures, typical of a dynamical instability: in some events fragments are formed very early or, in others, they can remain for a longer time attached to the leading PLF or TLF fragments [17-20]. Moreover, the projectile- and target-like primary fragments can be quite deformed at the rupture time and may follow a fast-fission path of purely dynamical nature [21]. For a given semi-peripheral impact parameter 
we expect to see a very wide velocity distribution in the fragment sources [17].

An intermediate behaviour between bulk and neck fragmentation, is observed for $b=4 \mathrm{fm}$ (fig.6). The freeze-out time is decreasing with impact parameter. This gradual transition suggests that it is quite inapproppriate to discuss all events in terms of a unique fragmentation mechanism and even harder to try to assign a fixed size or shape to a multifragmenting source, even passing from $b=2 \mathrm{fm}$ to $b=6 \mathrm{fm}$. Moreover the prompt nucleon emission should be always accounted for, at each step of the reaction dynamics.

A different aspect of the reaction mechanisms can be also seen from fig.7 where we show the parallel velocity distribution (in the $C M$ frame) of the produced fragments $(Z \geq 3)$ for semi-central (fig.7a), and semi-peripheral (fig.7b) collisions (neutron-rich case, asy-stiff EOS). In the semi-peripheral reactions (panel b) one can clearly distinguish the contributions of the spectators and the neck-IMF's. The slope of the spectator contributions is due to displaying the velocity rather than the momentum. A remnant of the spectator contribution is also seen in the semi-central events for $b=2 \mathrm{fm}$. Of particular interest is the larger velocity spread of the IMF's in the semi-peripheral relative to the semi-central events, which points to a different production mechanism. In general, the investigation of velocity distributions and correlations should yield further insight into the mechanism of the reactions, but we defer this to later work.

Guided by the density contour plots we have performed in the following a separate analysis of some quantities in a central region having a linear dimension of $20 \mathrm{fm}$ centered in the $C M$ of the system. Since this corresponds to the active volume in which fragmentation takes place (see figs. 5,6) we obtain in this way a more detailed picture of this process.

We organize the presentation of the results of the simulations in the following way. We first analyse the case of the $n$-rich reaction ${ }^{124} S n+{ }^{124} S n$. We study for the asy-stiff interaction the reaction mechanism for semi-central and semiperipheral collisions. Then we discuss the effects of different assumptions on the symmetry term. After that we discuss the differences for the $n$-poor case ${ }^{112} \mathrm{Sn}+{ }^{112} \mathrm{Sn}$.

\subsection{Results of ${ }^{124} \mathrm{Sn}+{ }^{124} \mathrm{Sn}$, $n$-rich case}

The results obtained with the asy-stiff symmetry term are shown in fig.8 (semicentral, $b=2 \mathrm{fm}$ ) and fig.9 (semi-peripheral, $b=6 \mathrm{fm}$ ). For each reaction the results are presented here and in the following cases in the following way:

Left column, time evolution of: (a) Mass in the liquid phase, $Z \geq 3$ (solid 

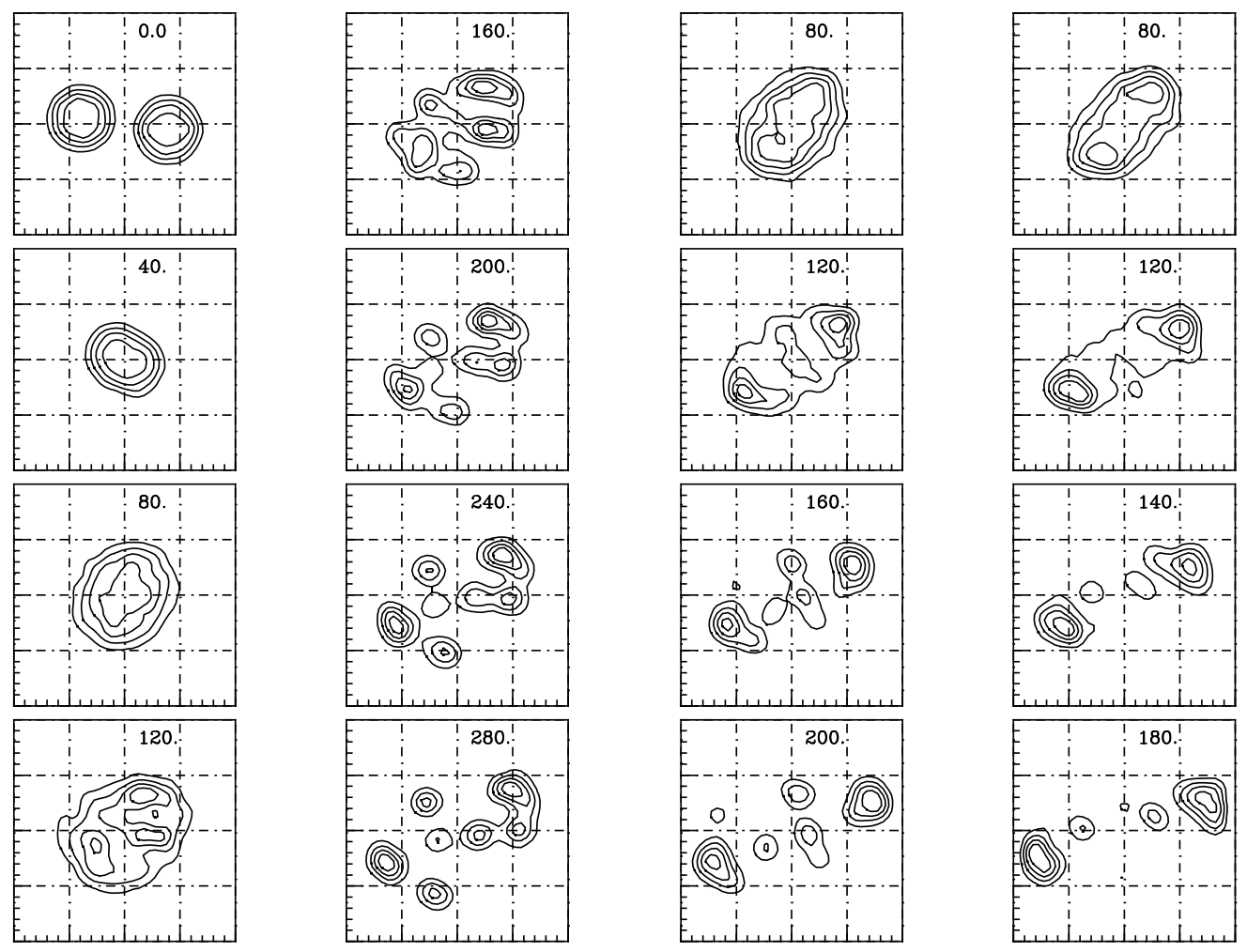

Fig. 5. ${ }^{124} S n+{ }^{124}$ Sn collision at $50 \mathrm{AMeV}$ : time evolution of the nucleon density projected on the reaction plane. Semi-central $b=2 \mathrm{fm}$ collision: approaching, compression, separation, and fragmentation phases. The times in $\mathrm{fm} / \mathrm{c}$ are written in each panel. The iso-density lines are plotted every $0.02 \mathrm{fm}^{-3}$ starting from $0.02 \mathrm{fm}^{-3}$.

Fig. $\quad 6 .{ }^{124} S n+{ }^{124}$ Sn collision at $50 \mathrm{AMeV}$ : time evolution of the nucleon density projected on the reaction plane, like in previous figure. First column: $b=4 \mathrm{fm}$, second column: $b=6 \mathrm{fm}$, separation phase up to the freeze-out.

line and dots) and the gas phase (solid line and squares); (b) Asymmetry $I=$ $(N-Z) /(N+Z)$ in the gas "central" (solid line and squares), gas total (dashed and squares), liquid "central" (solid and dots) phase and for IMF $(3<Z<15$, 

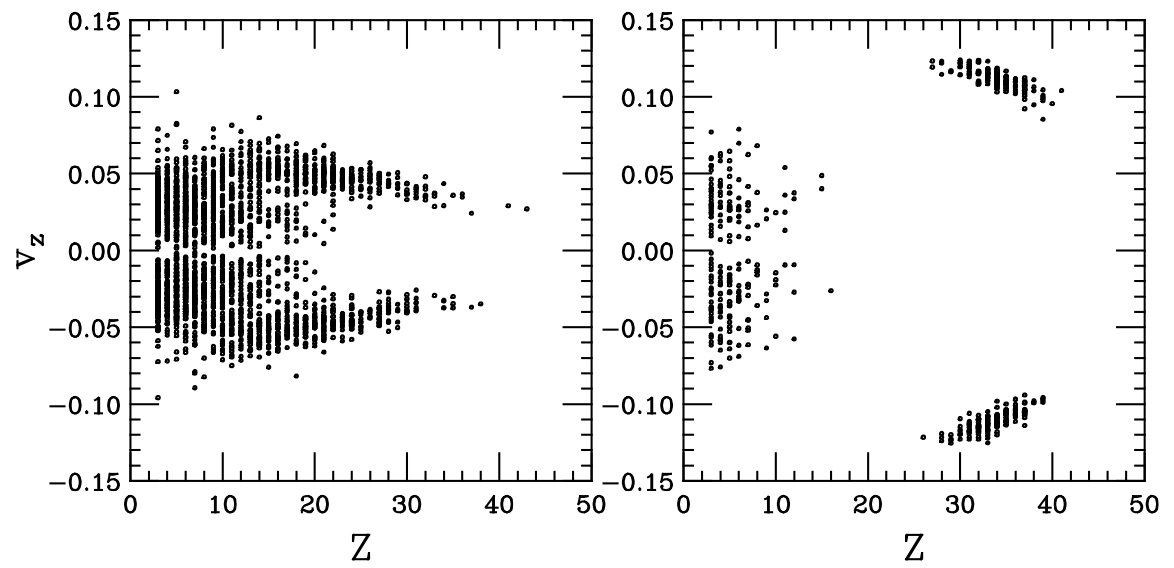

Fig. 7. ${ }^{124} \mathrm{Sn}+{ }^{124} \mathrm{Sn}$ collision at $50 \mathrm{AMeV}$ : parallel velocity distribution of all fragments $(Z \geq 3)$ at freeze-out time for an asy-stiff EOS: a) semi-central events $(b=2 \mathrm{fm})$; b) Semi-peripheral events $(b=6 \mathrm{fm})$. Asystiff EOS.

stars). The horizontal line shows the initial asymmetry; (c) Mean Fragment Multiplicity $Z \geq 3$. The saturation of this curve defines the freeze-out time and configuration. Right column, distributions of the "primary" fragments in the freeze-out configuration: (d) Charge distribution, (e) Asymmetry distribution as a function of the fragment charge and (f) Fragment multiplicity distribution (normalized to 1 ).

From these figures we can obtain a clear picture of the reaction mechanism. E.g. looking at fig. 8a we see the approach and compression phase up to about $50 \mathrm{fm} / \mathrm{c}$ in which almost all mass is still in the liquid, except a few pre-equilibrium particles. Between 50 and $120 \mathrm{fm} / \mathrm{c}$ we have the expansion phase in which many particles are emitted into the gas, but there is still one fragment (fig. 8c). In the fragmentation phase between 120 and $280 \mathrm{fm} / \mathrm{c}$ the number of fragments rises rapidly (fig. 8c), but fewer particles are emitted into the gas. The freeze-out time is characterized by the saturation of the number of fragments in fig. 8c. The same reaction phases are seen in the semiperipheral collision (figs. 9a,c), however, somewhat less destinct. The final charge distribution of semi-central and semi-peripheral collisions (figs. 8d and 9d) are distinctly different: in more central collisions it is rapidly decreasing, 

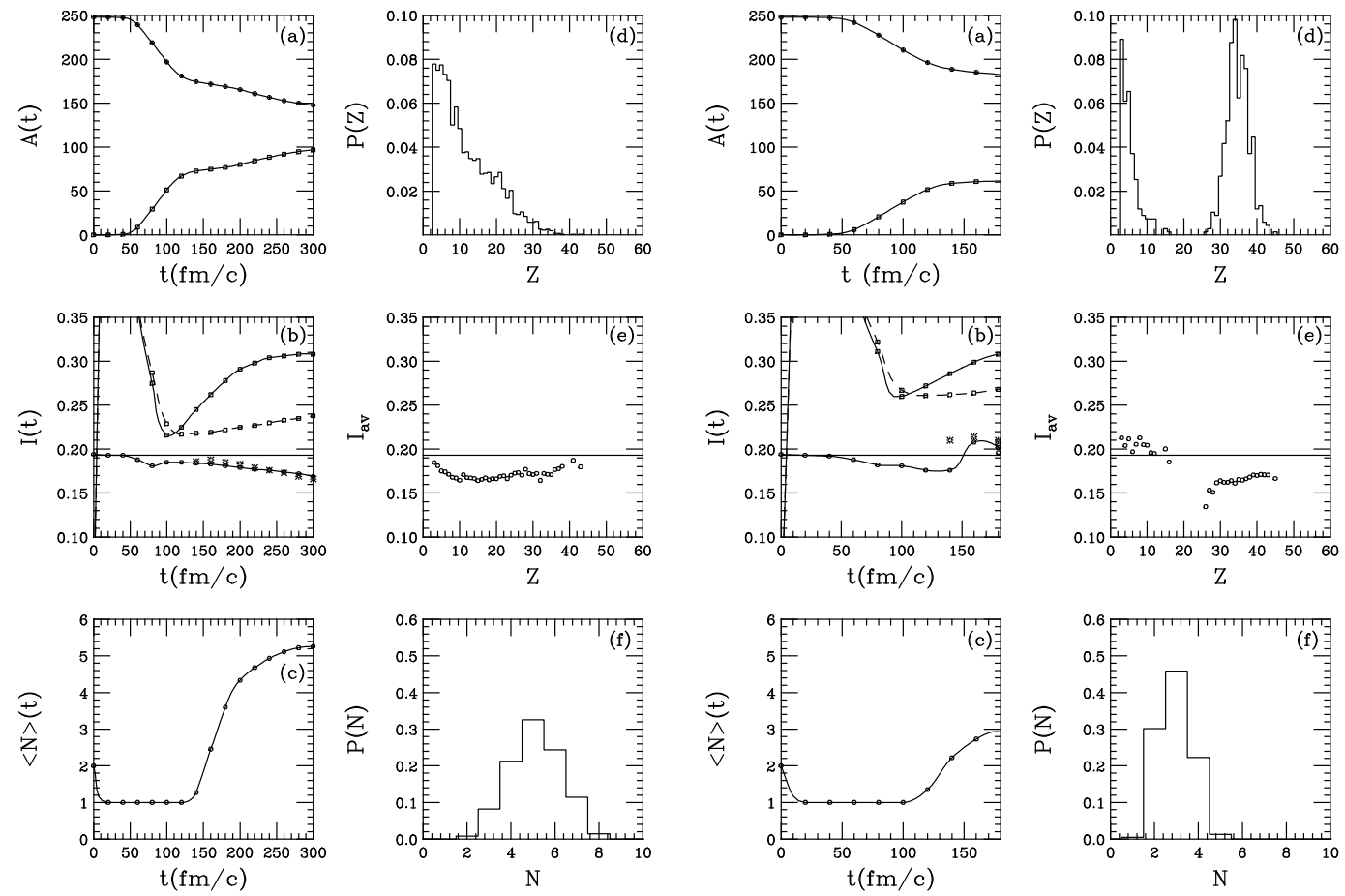

Fig. 8. ${ }^{124} \mathrm{Sn}+{ }^{124} \mathrm{Sn} b=2 \mathrm{fm}$ collision: time evolution (left) and freeze-out distributions (right), see text (ASY-STIFF EOS).

Fig. 9. ${ }^{124} \mathrm{Sn}+{ }^{124} \mathrm{Sn} b=6 \mathrm{fm}$ collision: time evolution (left) and freeze-out distributions (right), see text. ASY-STIFF EOS.

while in more peripheral collisions it shows a second maximum at the average charge of the spectators. Here the light fragments below charge 10 are the IMF produced in the neck. The average number of produced fragments is higher in the central case (figs. $8 \mathrm{c}$ and $9 \mathrm{c}$ at freeze-out times) and correspondingly the multiplicity of fragments is higher (figs. 8f and 9f). For the study of isospin dynamics the panels $b$ and $e$ are the most interesting, which give the evolution and final distribution of the isospin content of the various components in the reaction. They will be discussed below for the different reaction types. 


\subsubsection{Semi-central collisions: bulk fragmentation.}

We now discuss the isospin dynamics in the semi-central collisions, i.e. the behaviour given in figs. 8b,e. As seen the pre-equilibrium particles emitted during the compression phase are predominantly neutrons, as expected for the neutron-rich system. The particles emitted into the gas during the expansion phase consist equally of protons and neutron, thus lowering the asymmetry of the gas. The liquid phase becomes more symmetric during the compression due to neutron emission and then remains essentially constant during the expansion. At the beginning of the fragmentation phase, when density inhomogenities start to develop, an isospin burst of gas phase in the "central region" is observed (fig.8b) . At the same time the central liquid phase is becoming more and more symmetric. This behaviour is consistent with the dynamical spinodal mechanism in dilute asymmetric nuclear matter leading to isospin distillation.

In addition we observe very interesting features in the isospin content of the primary fragments in fig. 8e. We clearly see the isospin distillation effect, i.e. all IMF's are formed more symmetrically than the initial asymmetry. Moreover in fig.8e we can distinguish two opposite trends for fragments with charge above and below roughly $Z=15$. For the heavier fragments the average asymmetry increases with the charge. This is expected since for heavier nuclei a larger Coulomb effect must be compensated . However, the asymmetry rises again towards lighter fragments contrary to the trend for stable isotopes. We interpret this behaviour as a result of the different density regions in which fragments form and grow. The trend can be understood in a dynamical scheme following the transport effects of the symmetry term. This so-called proton migration effect, that yields more symmetric larger fragments, is seen more clearly in the more peripheral collisions, and will be discussed there.

However, as shown also in the density contour plot (fig.5) not all fragments form simultaneously, that makes the interpretation of the results not trivial.

\subsubsection{Semi-peripheral collisions: neck fragmentation.}

For semi-peripheral collisions at $b=6 \mathrm{fm}$ the isospin content appears with some new distinctive features as seen in fig. 9e. The IMF fragments formed in the neck region are much more asymmetric (more neutron rich) than the corresponding fragments produced in semi-central collisions (cf. fig. 8e). The heavy PLF and TLF have a definite lower asymmetry than the IMF's. We believe this to be due to a different nature of the fragmentation mechanism in the neck region, indicating a transition from volume to shape instabilities with a different isospin dynamics. To interpret this we have to keep in mind the following features of "neck-fragmentation": (i) The clusters are formed 
in a nuclear matter not very dilute relative to saturation density. We expect some density fluctuations but the fragmentation is mainly due to the shape dynamics [17]. (ii) The neck region is always in contact with a "high" density phase (the spectators) during the fragment formation. (iii) Due to a sponateous symmetry breaking of the neck instability in some events the fragments are formed closer to one of the spectators with increased interaction betweeen the two. Thus there will be a smooth transition to PLF/TLF fast-fission type of events [21].

Due to the increase of the symmetry term just below normal density it will be energetically more favorable to migrate protons from the neck region to the more dense spectators, leaving the nuclear matter in the neck more neutron rich at the time of breaking. In a sense the isospin dynamics is ruled by the same energetic argument as in the case of isospin distillation in central collisions. The main difference to the case of bulk fragmentation is now, that there is a spatial separation of the neutron-rich neck and the spectators, which initiates a flow of protons resp. neutrons in opposite directions. It thus involves an isovector mode of the mean field. Therefore we propose to call this phenomenon isospin migration in contradistinction to the distillation phenomenon, which is a spontaneous separation of the two phases.

Another difference is that we are testing the symmetry energy in different regions of nucleon density. In central collisions fragments are produced with the chemical instability mechanism in a very dilute asymmetric nuclear matter. The neutron distillation is therefore due to the symmetry energy increase at very low densities between 0.03 and $0.10 \mathrm{fm}^{-3}$ in our simulations. The neck matter, on the other hand, ruptures starting from densities just below the saturation values in contact with a normal density region given by the spectators. The symmetry energy range of interest here is now still in the subnuclear range but closer to $\rho_{0}$, between 0.08 and $0.16 \mathrm{fm}^{-3}$ in our simulations. This interpretation of the difference in the average isospin content of the fragments produced in semi-central and semi-peripheral collisions has a promising aspect: The sensitivity to the density dependence of the symmetry term in different regions of the subnuclear density range would allow the possibility of distinguishing between different effective interactions. This effect will be clearly seen below in our simulations.

The rise of the asymmetry of the IMF in semi-central collisions (fig. 8e) can also be interpreted as an isospin migration effect. In bulk fragmentation the smaller IMF's are also formed in contact with bigger fragments in the late stages of the process. Thus their isospin asymmetry increases by the same mechanism as that of neck fragments in peripheral colliions. 


\subsubsection{Symmetry term effects}

In this section we investigate the effects of different assumptions on the density dependence of the symmetry energy. In figs. 10,11 and 12,13 we report the results, obtained respectively with the asy-soft and the asy-superstiff symmetry term in the same format as was done for the asy-stiff symmetry term in figs. 8,9. The results for semi-central collision events, $b=2 \mathrm{fm}$, are thus in the figs. 8,10, and 12, while those for semi-peripheral collisions, $b=6 \mathrm{fm}$, are shown in figs. 9,11, and 13 .

As already observed the reaction dynamics is very rich. Thus also the fast particle emission in the expansion phase is affected by the symmetry term, in particular in the $N / Z$ composition. As seen before a large number of nucleons are emitted into the gas in the time interval between 50 and $120 \mathrm{fm} / \mathrm{c}$, i.e. in the expansion phase (see fig.5). This means that the symmetry part of the mean field at subnuclear densities will have an important dynamical effect on the emission. In the asy-soft case below $\rho_{0}$ neutrons are less bound than in the asy-superstiff case, and oppositely for the protons: we then expect a more neutron-rich prompt particle emission with the asy-soft symmetry term and a more symmetric initial dilute matter which will undergo fragment production. This is exactly what we see from the figs. 10b (asy-soft) and fig.12b (asysuperstiff) for central collisions. As expected the results of fig.8b (asy-stiff) are somewhat in between. It is interesting to remark that the same effect is present also for semi-peripheral collisions (compare figs. 11b and 13b), although more weakly.

We will now focus our discussion on the isospin content of the primary intermediate mass fragments $(3 \leq Z \leq 20)$. From a comparison of figs. 8e,10e and $12 \mathrm{e}$ for semi-central events and of figs. 9e,11e and 13e for semi-peripheral events we clearly see that the asysoft choice is the most effective for the neutron distillation effect (the most symmetric IMF's) in semi-central collisions while the asy-superstiff choice is the most effective in forming neutron-rich IMF's in the neck region for semi-peripheral collisions. Following the above discussion we easily understand these two trends considering the differences in the symmetry term at subnuclear densities (see figs. 1 and 2 of sect.2). The asy-soft parametrization gives a larger symmetry energy and a rather flat behaviour below normal density. Thus it is quite efficient during the chemical instability growth but not so for the proton migration from neck to spectators. Exactly the opposite trend is seen for the asy-superstiff parametrization with roughly a quadratic density dependence and thus a weak increase at low densities and a steep slope around $\rho_{0}$.

The differences between the results observed using different symmetry terms can also be interpreted in terms of the corresponding density behaviours of the $n, p$-chemical potentials, which were shown in fig. 3. As already noted above, 

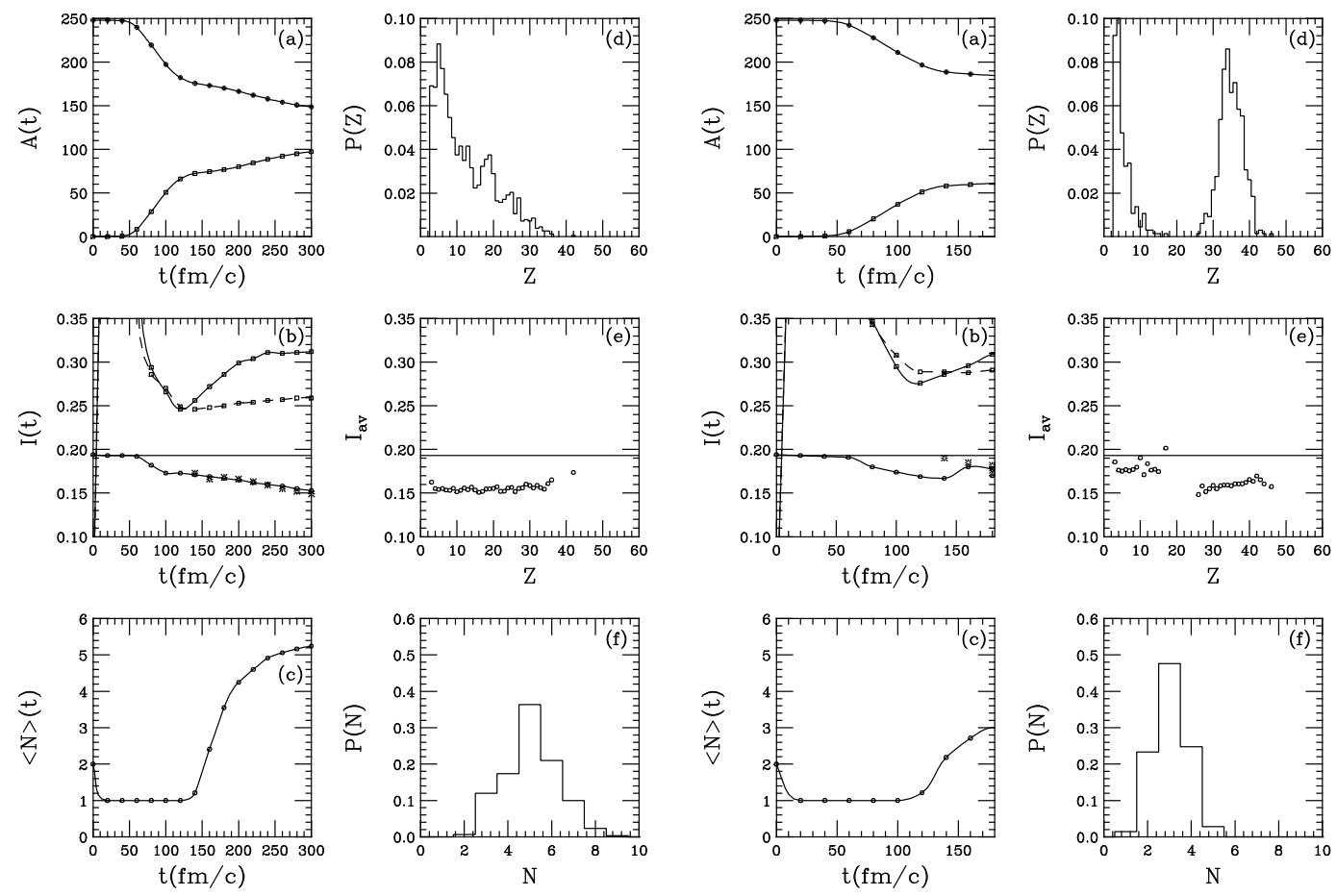

Fig. 10. ${ }^{124} \mathrm{Sn}+{ }^{124} \mathrm{Sn} b=2 \mathrm{fm}$ collision: time evolution (left) and freeze-out properties (right). See text. ASY-SOFT EOS

Fig. 11. ${ }^{124} \mathrm{Sn}+{ }^{124} \mathrm{Sn} b=6 \mathrm{fm}$ collision: time evolution (left) and freeze-out properties (right). See text. ASY-SOFT EOS.

we can distinguish two qualitatively different behavious in the subnuclear density region. At very low density, about below $0.08 \mathrm{fm}^{-3}$, i.e. in the region where bulk fragmentation and the isospin distillation effects take place both neutrons and protons have the tendency to move from lower to higher density regions. Since the variations of the two chemical potentials are different (larger for protons) we expect a lower asymmetry in the liquid phase [60] as discussed before. For neck fragmentation for a certain time interval we have contact between a more dilute phase (neck region) and the normal density regions of the PLT/TLF. This happens at density regions between $0.08 \mathrm{fm}^{-3}$ and 0.16 

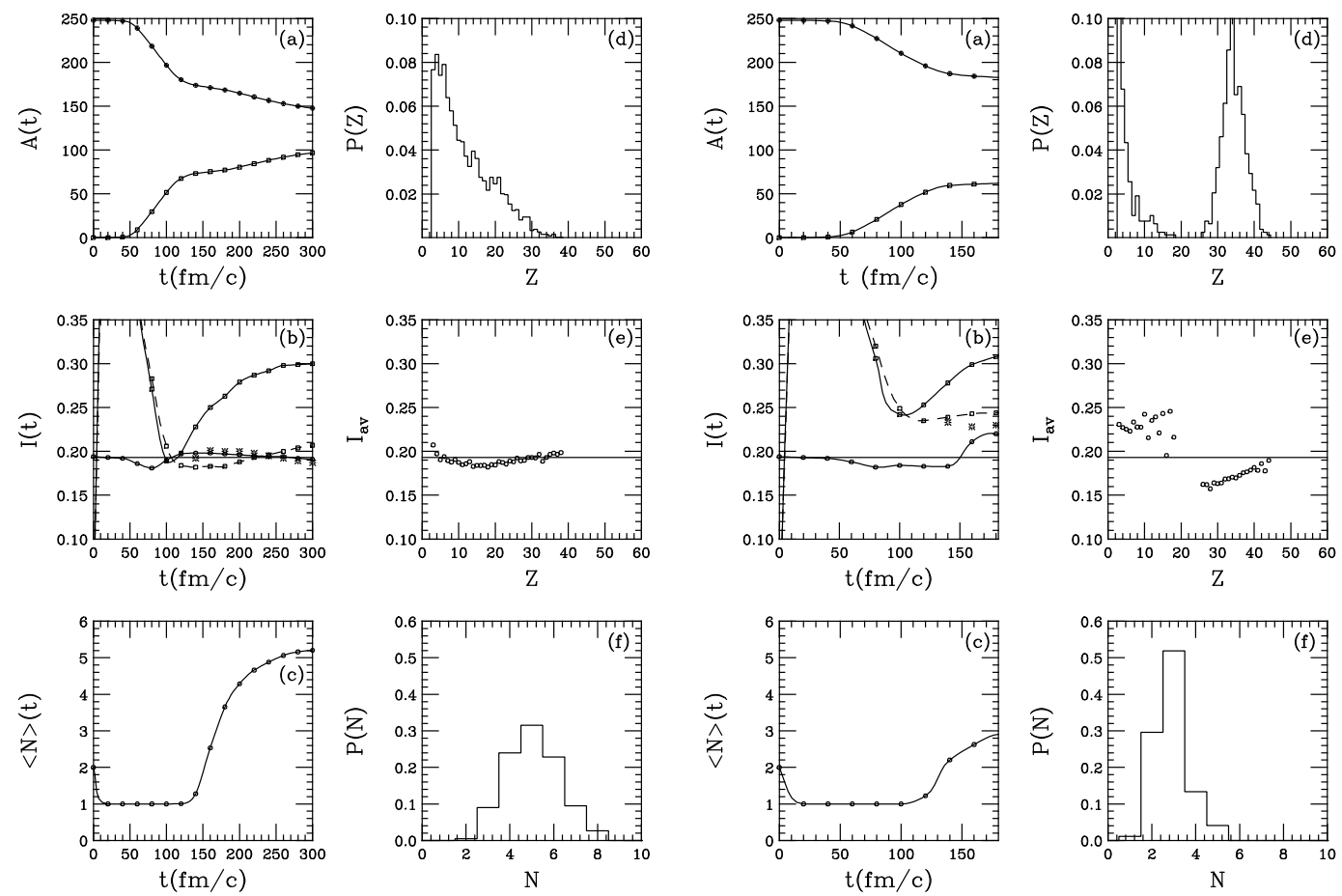

Fig. 12. ${ }^{124} \mathrm{Sn}+{ }^{124} \mathrm{Sn} \quad b=2 \mathrm{fm}$ collision: time evolution (left) and freeze-out properties (right). See text. $A S Y$-SUPERSTIFF EOS

Fig. 13. ${ }^{124} \mathrm{Sn}+{ }^{124} \mathrm{Sn} \quad b=6 \mathrm{fm}$ collision: time evolution (left) and freeze-out properties (right). See text. $A S Y$-SUPERSTIFF EOS.

$\mathrm{fm}^{-3}$, where we see from fig. 3 that neutrons have the tendency to move toward more dilute regions producing a $n$-enrichment of the neck, while protons will migrate towards the higher density regions of the PLF/TLF. This explains the large difference in asymmetry between the PLF/TLF and the neck fragments. Moreover the neck IMF's will be always more $n$-rich compared to the fragments produced in the case of bulk fragmentation. The relative motion of the spectators as well as surface instabilities and Coulomb effects will impose some limitations to this interpretation, but it seems to give the main features and a consistent picture of the results obtained in simulations. 
It should be noticed that this effect is in addition to the geometrical neutron enrichment just coming from the way the neck region originates. In fact the overlap of the surface of PLF and TLF naturally yields a neutron-rich region, due to the neutron skin. This effect should be larger in the stiff parameterizations where, due to the steep behavior around normal density, a larger neutron skin is predicted.

Above we have discussed the relation between the average $N / Z$ of the IMF's produced in the neck region and the slope of the symmetry term, i.e. the symmetry pressure, just below normal density. A similar information is contained, in a more averaged way, in the fragment multiplicity distributions (figs. 9f,11f, and 13f). Multi neck-IMF events are decreasing with increasing stiffness of the symmetry term: comparing figs. $11 \mathrm{f}$ and $13 \mathrm{f}$ we note that in the asy-superstiff case we have $\simeq 30 \%$ more binary events. This is indeed a signature of the larger repulsion in the neck zone due to the larger symmetry pressure. Thus even a very inclusive observable, namely fragment multiplicity, appears to be sensitive to the symmetry term for semi-peripheral collisions. We note that the slope of the symmetry energy around normal density is exactly the symmetry pressure we expect to see active in the neutron rich surface of heavy elements, which is thus of large importance in determining the difference between matter and proton distributions (see the recent discussion in refs. $[10,11])$. Of course, the question is absolutely critical for the discussion of unstable nuclei and neutron skins and halos. We thus see the complementarity between reaction and structure studies.

\subsection{Results of ${ }^{112} S n+{ }^{112} S n, n$-poor case}

The results for collisions of the neutron-poor system ${ }^{112} S n+{ }^{112} S n$ are shown in the same format as before for the asy-stiff choice in figs. 14,15 and for the asy-soft choice in figs. 16,17. Since in this system the initial asymmetry is on the $p$-rich side of the valley of stability there is a general trend of the fragments to move towards stability, i.e. to asymmetries which are larger than the inital one. This is contrast to the $n$-rich case of ${ }^{124} S n$ where the general trend was towards fragments which are more symmetric than the initial asymmetry. On this general trend are superimposed the mechanisms of isospin distillation and migration in a similar way as in the $n$-rich case. Thus asymmetry distributions roughly appear just shifted with respect to the $n$-rich case (cf. e.g. figs. 14b,15b with figs. 8b,9b). In particular, we notice again a larger asymmetry of the IMF's emitted from the neck in the asy-stiff case owing to a more efficient simultaneous migration of protons towards the denser regions of the PLF/TLF and of neutrons in the opposite direction.

A qualitative difference relative to the $n$-rich system is a larger prompt proton 

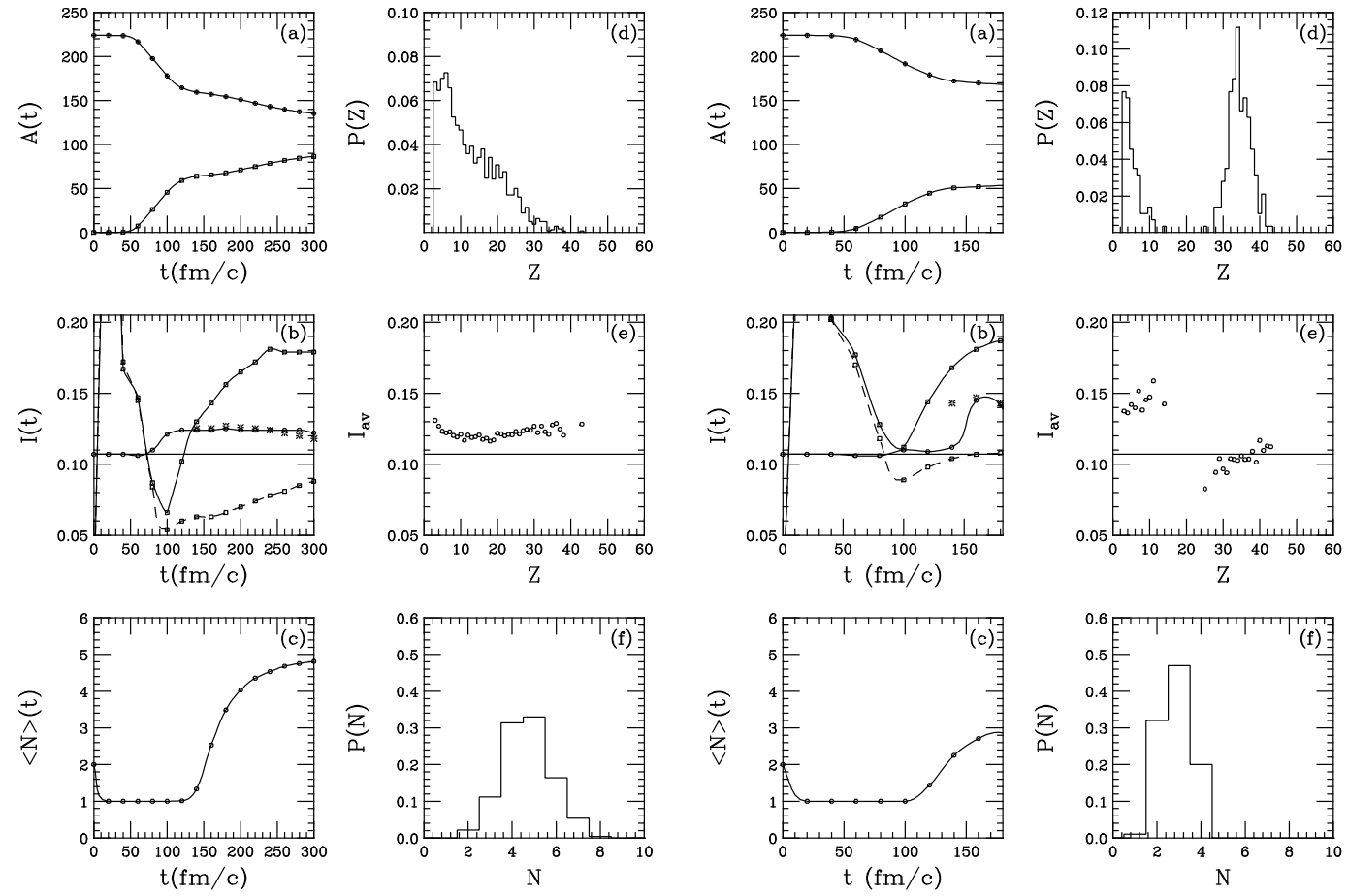

Fig. 14. ${ }^{112} S n+{ }^{112}$ Sn $b=2$ fm collision: time evolution (left) and freeze-out properties (right). See text. ASY-STIFF EOS

Fig. 15. ${ }^{112} \mathrm{Sn}+{ }^{112} \mathrm{Sn} b=6 \mathrm{fm}$ collision: time evolution (left) and freeze-out properties (right). See text. ASY-STIFF EOS.

emission during the expansion phase, in particular for semi-central collisions as we see from the time evolution of gas and liquid asymmetry (figs.14b,16b). Apart from the Coulomb repulsion the protons are less bound at subnuclear densities (fig.2). This effect is larger for a stiffer symmetry term, as seen by comparing the gas asymmetry evolution in figs. 14b and 16b. Thus when the liquid phase is breaking up it has a larger asymmetry than the initial one and we see an isospin distillation effect, which is almost absent in the asy-soft case (fig.16e).

In the asy-soft case the neck isospin enrichment is produced mainly by the neutron migration from the PLF/TLF while the proton drift is very reduced 

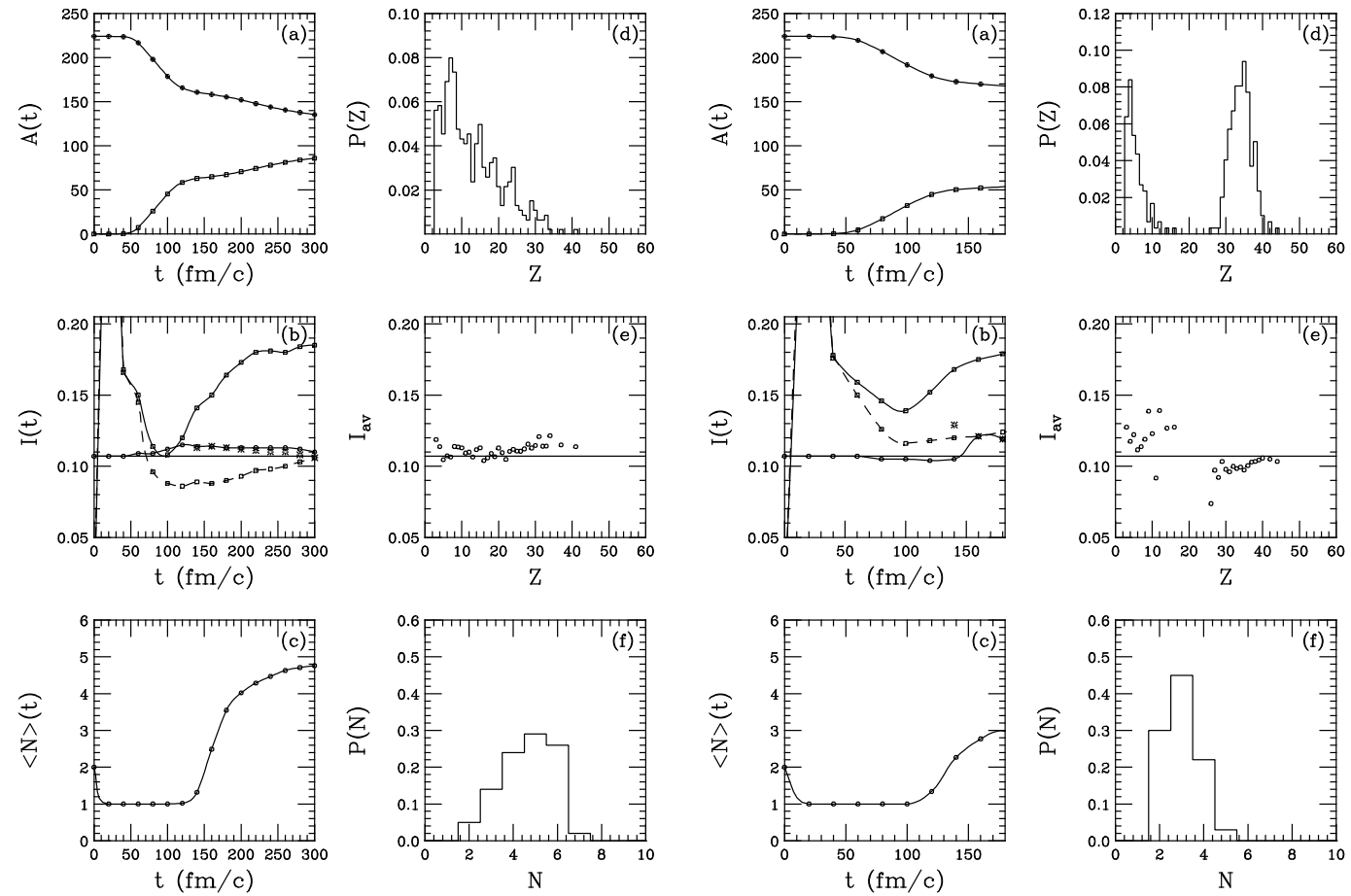

Fig. 16. ${ }^{112} S n+{ }^{112}$ Sn $b=2$ fm collision: time evolution (left) and freeze-out properties (right). See text. ASY-SOFT EOS

Fig. 17. ${ }^{112} \mathrm{Sn}+{ }^{112} \mathrm{Sn} b=6 \mathrm{fm}$ collision: time evolution (left) and freeze-out properties (right). See text. ASY-SOFT EOS.

between 0.08 and $0.15 \mathrm{fm}^{-1}$ densities (see lower dashed curve of fig.3). Therefore more protons remain in the low density region, the fragments are less asymmetric, and the difference relative to the PLF/TLF asymmetry is reduced (compare figs.15e and 17e).

We observe an interesting effect of the symmetry term on the IMF multiplicity in the bulk fragmentation. In fig. 18 we show the average IMF multiplicity vs. the average charge of the heaviest produced fragment, which is a measure of the centrality of the collision, for two symmetry terms and for the two systems, $n$-rich and $n$-poor. A difference between the multiplicities for the two systems is evident for the asy-stiff case in semi-central collisions (low $<Z>_{\text {heavy }}$ ). 
For the asy-soft case the difference is very reduced. We ascribe the effect to the fact that with a stiffer symmetry term protons in a n-rich system are very efficiently used to form clusters, see the chemical potential argument. Such differences have actually been observed in recent experimental data [61], which could be an indication for a symmetry term of asy-stiff type.

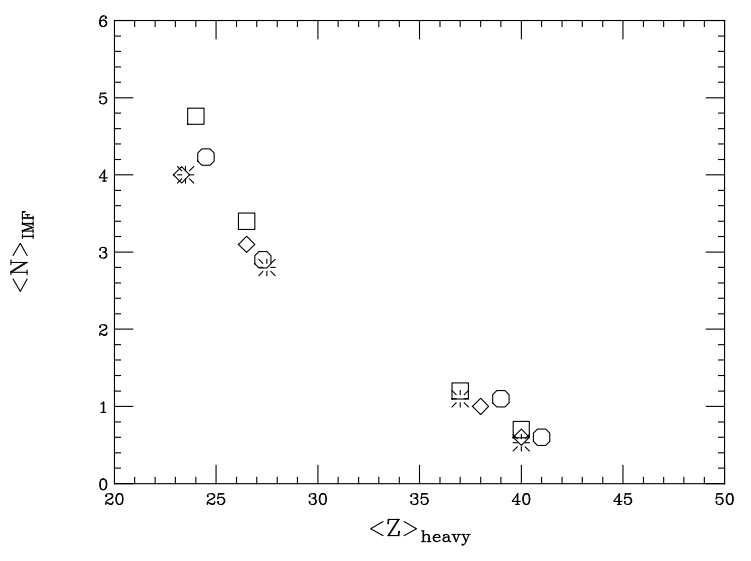

Fig. 18. Correlation between mean IMF multiplicity and charge of the heaviest fragment: (squares) n-rich "asy-stiff"; (diamonds) n-poor "asy-stiff"; (circles) n-rich "asy-soft"; (stars) n-poor "asy-soft".

We note that more definitive conclusions can be obtained from precise $N / Z$ measurements of the emitted IMF's with a good selection of the sources. A larger absolute value of neck-IMF asymmetry in comparison with that of fragments produced in semi-central collisions as well as a large difference relative to that of the PLF/TLF source will strenghten the above indication.

\section{Outlook}

In this paper we have tried to make a connection between chemical instabilities in an infinite binary system of protons and neutrons and isospin transport properties in microscopic simulations of fragmentation reactions of charge 
asymmetric ions in the medium energy range between 20 and $100 \mathrm{AMeV}$. We have investigated observables that seem to be rather sensitive to the symmetry term of the nuclear EOS. A first conclusion of the detailed analysis of our dynamical simulations is that in this energy range we expect to see a characteristic behaviour of the $N / Z$ of the intermediate mass fragments as a function of the centrality of the reaction, a typical rise and fall with decreasing impact parameter. For peripheral collisions IMF will be emitted in a statistical way from the excited PLF/TLF regions close to the stability line. For semi-peripheral events the neck-fragmentation mechanism will form more neutron-rich fragments from dynamical effects. For central collisions the neutron distillation will take place and fragments will be again much more symmetric. The relevance of such behaviour is related to the stiffness of the symmetry term at subnuclear densities, and this could be a very important information to extract from fragmentation data, provided a very accurate centrality selection of the events is performed.

The proton fraction of fast particle emission appears to be quite sensitive to the slope of the symmetry term around normal density. For central collisions this effect introduces the observed difference in IMF's multiplicities between $n$-rich and $n$-poor systems.

Isospin migration in the "neck instabilities" will also induce interesting isotopic effects on the expected fast-fission breaking of the projectile-like and/or targetlike fragments.

Of course all the results discussed here refer to the primary fragments, i.e. at the freeze-out time. Of course these are well excited and the subsequent statistical decay can modify the signal. Some of the effects discussed here appear to be quite robust and indeed from the first available data the isospin dependence of fragment production emerges quite clearly. However, a detailed investigation of the secondary decay, which depends crucially on the isospin content and the excitation energy of the primary fragments is clearly neccessary, in order to make connections to experimental observations.

Moreover we have not presented here more detailed dynamical properties of the fragment emission, like velocity distributions and correlations, kinetic and excitation energies, angular distributions, collective flows, etc. The present results are already very promising and we can be confident that with more exclusive data and more asymmetric (also radioactive) beams we will be able to perform detailed studies of the "elusive" symmetry term of the nuclear EOS. 


\section{Acnowledgements}

We gratefully acknowledge intense and stimulating discussions with several people actively working on isospin effects in nuclear dynamics. In particular we would like to mention Bao-An Li, I.Bombaci, Ph.Chomaz, I.Hamamoto, H.Horiuchi, U.Lombardo, A.Ono, U.Schroeder, L.Sobotka and S.Yennello. Special thanks are due to the experimental groups working at the NSCL-MSU (W.G.Lynch, M.B.Tsang, G.Verde and R. de Souza) and at the CS-LNS (A.Pagano, E.Geraci, E.Piaceski and J.Wilczinsky) for the prompt availability of their inspiring new $4 \pi$ data. 


\section{References}

[1] I.M.Irvine, "Neutron Stars" Oxford Univ. Press 1978

[2] J.M.Lattimer et al., Phys.Rev.Lett. 66 (1991) 2701

[3] D.Pines, R.Tamagaki and S.Tsuruta Ed.s, "Neutron Stars", Addison-Wesley N.Y.1992

[4] K.Sumiyashi and H.Toki, Astro.Phys.Jour. 422 (1994) 700

[5] C.J.Pethick and D.G.Ravenhall in "The Lives of Neutron Stars" Ed.s M.A.Alpar et al., NATO ASI Series C Vol.450 (1995) pp.59-70

[6] C.H.Lee, Phys.Rep. 275 (1996) 255

[7] I. Bombaci, T.T.S. Kuo and U. Lombardo, Phys. Reports. 242 (1994) 165. I. Bombaci, Phys. Rev. C55 (1997) 1

[8] M.Prakash et al., Phys.Rep.280 (1997) 1

[9] "It is important to put the properties of neutron matter at subnuclear densities on as firm a footing as possible, not only for astrophysical applications, but also for interpreting terrestrial experiments with coming radioactive beam facilities" C.J. Pethick and D.G. Ravenhall, in ref.[5].

[10] B.A.Brown, Phys.Rev.Lett. 85 (2000) 5296

[11] C.J.Horowitz and J.Piekarevicz, Phys.Rev.Lett. 86 (2001) 5647.

[12] H.Mueller and B.D.Serot, Phys.Rev. C52 (1995) 2072

[13] Bao-An Li and C.M.Ko, Nucl.Phys. A618 (1997) 498

[14] M.Colonna, M.Di Toro and A.Larionov, Phys.Lett. B428 (1998) 1

[15] V.Baran, M. Colonna, M. DiToro and V.Greco, Phys.Rev.Lett. 86 (2001) 4492

[16] M.Colonna, M.DiToro, G.Fabbri and S.Maccarone, Phys.Rev. C57 (1998) 1410

[17] M.Colonna, M.Di Toro and A.Guarnera, Nucl.Phys. A589 (1995) 160;

J.D.Frankland, Thesis Orsay 1998;

[18] J.Toke et al., Phys.Rev.Lett. 77 (1996) 3514 and ref.s therein.

[19] D.Durand, Nucl.Phys. A654 (1998) 273c

[20] E.Plagnol et al.(INDRA collab.), Phys.Rev. C61 (2000) 014606

[21] F.Bocage, J.Colin et al., Nucl.Phys. A676 (2000) 391

[22] G.Westfall, Nucl.Phys. A630 (1998) 27c

[23] Bao-An Li, C.M.Ko and W.Bauer, Int.J.Mod.Phys. E7 (1998) 147-229 
[24] M.Di Toro et al., Progr.Part.Nucl.Phys. 42 (1999) 125-136

[25] L.G. Sobotka et al., Phys. Rev. C55 (1997) 2109, and in "Multifragmentation", Ed.s H. Feldmeier, J. Knoll, W. Noerenberg and J. Wambach, GSI 1999, p.7281.

[26] H.Xu et al., Phys.Rev.Lett. 85 (2000) 1908

[27] G.Verde et al., Nucl.Phys. A681 (2001) 299c

[28] M.B.Tsang et al., Phys.Rev.Lett. 86 (2001) 5023, W.P.Tan et al. Fragment isotope distributions and the isospin dependent EOS, arXiv:nucl-ex/0104017.

[29] S.J.Yennello et al., Nucl.Phys. A681 (2001) 317c

[30] W.U.Schroeder et al., Nucl.Phys. A681 (2001) 418c

[31] E.Geraci, A.Pagano et al. (Chimera Collab.), isospin data from the ${ }^{124} \mathrm{Sn}+{ }^{64} \mathrm{Ni}$ at $35 \mathrm{AMeV}$, private communication

[32] R.de Souza et al., Isospin data from the ${ }^{114} \mathrm{Cd}+{ }^{92} \mathrm{Mo}$ at $50 \mathrm{AMeV}$, private communication and H.Xu et al. Fragment Isospin as a Probe of Heavy-Ion Collisions, arXiv:nucl$\mathrm{ex} / 0107012$

[33] Bao-An Li, Phys.Rev.Lett. 85 (2000) 4221

[34] M.DiToro et al., Nucl.Phys. A681 (2001) 426c

[35] H.Krivine, J.Treiner and O.Bohigas, Nucl.Phys. A336 (1980) 155

[36] E.Chabanat, P.Bonche, P.Haensel, J.Meyer, R.Schaeffer, Nucl.Phys. A627 (1997) 710

[37] We remark here that also all relativistic mean field approaches in the presently used approximation scheme are predicting a stiff-like simmetry term, proportional to the barion density, coming from the $\rho$-meson field contribution, see ref.[38]. Actually a superstiff behaviour is obtained when a coupling to the scalar charged meson $\delta$ is added, see ref.s $[39,40]$.

[38] S.Yoshida, H.Sagawa and N.Takigawa, Phys.Rev. C58 (1998) 2796

[39] S.Kubis and M.Kutschera, Phys.Lett.B399 (1997) 191;

[40] V.Greco et al., Asymmetric nuclear matter in a Hatree-Fock approach to non linear quantum hadrodynamics, arXiv:nucl-th/001133, Phys.Rev.C in press

[41] V.Baran, M.Colonna, M.Di Toro, and A.Larionov, Nucl.Phys. A632 (1998) 287

[42] L.D. Landau and E.M. Lifshitz, Statistical Physics, Pergamon Press, pag. 208.

[43] G. Baym and C. Pethick, Landau Fermi-liquid theory (John Wiley and Sons, New York, 1991) 
[44] J.P. Bondorf, A.S. Botvina, A.S. Iljinov, I.N. Mishustin, K. Sneppen, Phys. Rep. 257 (1995) 133.

[45] A.B.Larionov,A.Botvina,M.Colonna and M.DiToro, Nucl.Phys. A658 (1999) 375

[46] G.F.Bertsch and S.Das Gupta, Phys.Rep. 160 (1988)189

[47] Ch.Gregoire et al., Nucl.Phys. A465 (1987) 315

[48] A.Bonasera et al., Phys.Rep. 244 (1994) 1

[49] A.Bonasera, G.F.Burgio and M.DiToro, Phys.Lett. B221 (1989) 233 and A.Bonasera and F.Gulminelli, Phys.Lett. B259 (1991) 399

[50] TWINGO code: A.Guarnera Ph.D.Thesis, Univ.Caen , July 1996

[51] L.Scalone, M.Colonna and M.DiToro, Phys.Lett. B461 (1999) 9

[52] M.Colonna, m. DiToro, A. Guarnera, S. Maccarone, M. Zielinska-Pfabe, H.H. Wolter, Nucl.Phys. A642 (1998) 449

[53] V.Greco, A.Guarnera, M.Colonna and M.DiToro, Phys.Rev. C59 (1999) 810

[54] S. Ayik and C. Gregoire, Phys. Lett. B212 (1988) 269; Nucl. Phys. A513 (1990) 187.

[55] J. Randrup and B. Remaud, Nucl. Phys. A514 (1990) 339.

[56] M.Colonna and Ph.Chomaz, Phys.Rev. C49 (1994) 1908;

Ph.Chomaz, M.Colonna, A.Guarnera and J.Randrup, Phys. Rev. Lett. 73 (1994) 3512.

[57] M. Colonna, G.F. Burgio, Ph. Chomaz, M. Di Toro, and J. Randrup, Phys. Rev. C47 (1993) 1395;

[58] M. Colonna, M. Di Toro and A. Guarnera, Nucl. Phys. A580 (1994) 312.

[59] A. Guarnera, M. Colonna and Ph. Chomaz, Phys. Lett. B373 (1996) 267; M. Colonna, Nucl. Phys. A630 (1998) 136c.

[60] We like to note that the argument is valid not only inside the spinodal region where the nuclear phase is unstable but also in metastability regions were the liquid phase is developing from an initial homogeneous gas via "nucleation" due to large amplitude fluctuations. So we expect isospin distillation also in the much slower nucleation mechanism.

[61] M.L.Miller et al., Phys.Rev.Lett. 82 (1999) 1399 and ref.s therein. 